\title{
Cat Inflicted Eye Injury in a Child
}

\author{
Deeksha Borkar ${ }^{\mathrm{a}, \mathrm{c}}$, Pablo Avendano ${ }^{\mathrm{b}}$
}

\begin{abstract}
Ocular injuries, especially corneal abrasions are a common phenomenon noted in the pediatric emergency department. Most of these are minor injuries and are well described in literature. However, there is very limited data on corneal cat scratch injuries and their management. We present the case of a 10-year-old female with cat scratch inflicted superficial corneal laceration. In addition to immediate identification of the injury, it is essential to investigate and evaluate the depth of the ocular injury for prompt management, including route of antibiotics, need for an immediate surgical intervention and future follow-up.
\end{abstract}

Keywords: Corneal abrasion; Cat scratch; Ocular injuries; Emergency

\section{Introduction}

Ocular injuries, especially corneal abrasions are a common phenomenon noted in the pediatric emergency department and urgent cares. Corneal abrasion can present with a wide variety of symptoms including excessive tearing, foreign body sensation, photophobia, conjunctival injection and so on [1]. Detailed examination of the eye with fluorescein staining and ultraviolet light is the standard of care. Treatment is dependent on the mechanism and extent of injury. In most cases of corneal abrasion, treatment comprises of pain control topical antimicrobial prophylaxis and close follow-up care [2].

Most of these corneal abrasions are minor injuries, occur accidently and are well described in literature. However, there is very limited data on corneal cat scratch injuries and their management. We present the case of a 10-year-old female with cat scratch inflicted superficial corneal laceration. In addition

Manuscript submitted January 26, 2021, accepted February 9, 2021

Published online March 5, 2021

aDepartment of Pediatrics, University of Minnesota Medical School, Minneapolis, MN, USA

${ }^{b}$ Division of Pediatric Emergency Medicine, Department of Pediatrics, University of Minnesota Medical School, Minneapolis, MN, USA

${ }^{\mathrm{c} C}$ Corresponding Author: Deeksha Borkar, Department of Pediatrics, University of Minnesota Medical School, 2450 Riverside Avenue, Room M136, Minneapolis, MN 55454, USA. Email: borka015@umn.edu

doi: https://doi.org/10.14740/jmc3663 to immediate identification of the injury, it is essential to investigate and evaluate the depth of the ocular injury for prompt management, including route of antibiotics, need for an immediate surgical intervention and future follow-up.

\section{Case Report}

A 10-year-old female presented to the pediatric emergency department approximately $2 \mathrm{~h}$ after she sustained a cat claw scratch to her right eye by her pet cat. Initially the patient informed her mother that she tripped and fell, and her eye was painful. The parent offered some ice packs. As the patient continued to have persistent pain and blurry vision, she informed her mother of the increasing pain. The patient's mother immediately examined her eye with a flashlight and noted a scratch on the cornea. The patient then disclosed to her mother the events that occurred, and the patient's mother decided to bring her to the pediatric emergency department for further evaluation.

At the emergency department triage, the patient did endorse epiphora of the right eye and blurry vision but denied any pain on eye movement. On physical exam, the patient was alert, breathing comfortably and in mild pain. The patient was able to actively open both eyes and did not have any pain on extra ocular movements. The conjunctiva of the right eye was mildly injected with minimal clear watery discharge. No purulent discharge was noted on examination. The pupils were symmetric, round and reactive to light and accommodation. The left eye was normal without any conjunctival injection.

Visual acuity for the left eye was 20/25 and for the right eye was 20/32. For a detailed eye exam, topical anesthesia with proparacaine hydrochloride $0.5 \%$ ophthalmic solution was instilled near the inner canthus of the right eye. A fluorescein examination of the right eye was indicative of immediate dye uptake (Fig. 1). Examination of the right eye under ultraviolet light revealed a single, diagonal and linear corneal laceration of approximately $5 \mathrm{~mm}$ in length extending from the superior portion of the cornea up to the inferior limbus. A Seidel test was negative. A slit-lamp examination and tonometry were not performed. A corneal culture was not obtained.

An ophthalmology consulting was requested in the light of a cat scratch inflicted corneal laceration and the possibility of infectious keratitis. Immunization review of the patient revealed a missing tetanus booster. The patient received Tdap immunization in the emergency department. Rabies risks were dismissed due to the fact that the pet was an indoor cat. The pa- 


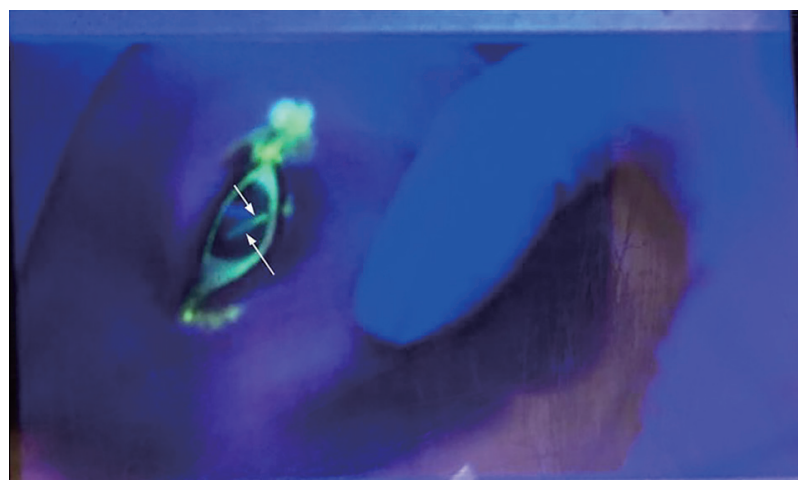

Figure 1. A fluorescein examination of the right eye. Arrows indicate fluorescein staining of the cornea visualized under ultraviolet light and a linear corneal laceration.

tient was discharged with a diagnosis of corneal laceration and prescriptions of moxifloxacin ophthalmic solution $0.5 \%$ and polyvinyl alcohol (liquifilm tears) $1.4 \%$ ophthalmic solution to be instilled in the right eye four times daily. The patient's mother was advised to initiate treatment at the earliest and recommended to follow up with the ophthalmologist in 3 days for a follow-up eye exam. Precautions and reasons to return were discussed with the patient's parent.

Follow-up exam was indicative of improvement in visual acuity with left eye acuity at 20/20 and right eye at 20/15. Foreign body sensation decreased, and eye drops were discontinued. Importance of safety around the family cat was discussed with the patient and parents in detail.

\section{Discussion}

Cat scratch inflicted corneal injuries may produce serious eye trauma with a spectrum from corneal abrasion to globe rupture, infections caused by Bartonella henselae, Pasteurella multocida and life-threatening conditions caused by tetanus and rabies.

Case reports of cat scratch inflicted corneal lacerations are seldom described in literature. In the pediatric emergency department and urgent care settings, cat inflicted eye injuries are also fairly rare with the spectrum of injuries ranging from a simple abrasion to globe rupture in the few cases that have been reported [3, 4]. In our case, we report a new and unique case of cat scratch inflicted corneal laceration and approach to emergent care and antimicrobials. As exemplified in our case, cat scratch infected corneal abrasions and lacerations are uncommon presentations in the emergency and urgent care settings. Prompt medical attention and evaluation are essential for prevention of vision loss. Immediate recognition of the potential for serious corneal or globe damage with the potential of secondary microbial infection is important for practitioners for clinical decision-making regarding choice and route of antibiotics.

In 2012, a case series and literature review of cat scratch inflicted corneal injuries described three cases at tertiary care centers in Australia with varied extents of injury and antimicrobial management [4]. All the three cases had excellent visual outcomes with different approaches of management. In all cases, identification of the extent of injury was the key to management and antimicrobial approach. The Seidel test, a test that detects the presence of aqueous humor leakage from the anterior chamber, is critical in identifying penetrating eye injuries [5]. In all cases, once the injury is identified, it is of vital importance to provide prompt surgical or medical management depending upon the depth of the penetrating scratch laceration. Most superficial corneal lacerations are treated with topical antimicrobials and lubricating ointments in combination [6]. However, in the case of cat scratch inflicted eye injuries, it is important to identify the potential organisms to direct antimicrobial choice. Bartonella henselae is most commonly the cause of cat scratch disease [7]. Pasteurella multocida, a Gram-negative coccobacillus is also a commonly associated microflora with most mammals including cats and dogs [8]. Few cases of Pasteurella multocida endophthalmitis have been described including most recently after a cat scratch in a 10-year-old girl in Japan [9]. Pasteurella multocida and Bartonella henselae are both Gram-negative organisms and susceptible to penicillins, cephalosporins, tetracyclines, macrolides and fluoroquinolones among others [10-13].

Along with Pasteurella multocida and Bartonella henselae, there are two other infectious organisms that are seen in animal inflicted injuries, namely Clostridium tetani and rabies lyssavirus [14]. Rabies is a zoonotic infection caused by the rabies lyssavirus, belonging to the genus lyssavirus. According to the Centers for Disease and Control (CDC), cases of human rabies are rare in the United States and the number of human rabies deaths have been gradually declining due to animal control and vaccination programs [15]. Tetanus disease is caused by an extremely potent neurotoxin released by the bacteria Clostridium tetani [16].

Tetanus immunization is routinely recommended to all individuals after an animal inflicted injury who have not received a booster vaccine for more than 5 years prior to injury $[17,18]$.

In most cases of corneal abrasions, the pediatric emergent and urgent medicine management approach comprises of erythromycin ophthalmic ointment for 3 days and follow-up with a primary care provider if needed. Erythromycin eye ointment, along with its antimicrobial activity, serves the dual purpose of a lubricant and protects the process of epithelialization along with its antimicrobial protection [19].

In our case, as the depth of the laceration was superficial, a choice of moxifloxacin ophthalmic solution $0.5 \%$ was made due to its high lipophilicity, better corneal penetration and tissue concentrations, broad spectrum activity and high aqueous solubility at physiologic eye $\mathrm{pH}[20,21]$. In full thickness corneal lacerations, choice and route of antibiotics may differ. Systemic, intravitreal or intracameral antibiotics with surgical management may be used depending on the presentation, duration, location and extent of the laceration. The goal of the antimicrobials in cat scratch inflicted corneal lacerations is to provide adequate protection against secondary microbial keratitis and prevention of permanent vision loss.

In addition to antimicrobials, it is important to initiate the use of artificial tears as a form of lubrication to provide comfort and promote reepithelialization [22]. Most artificial tears 
are available over the counter and may contain one or more of the following ingredients: polyvinyl alcohol, polyethylene glycol, carboxymethylcellulose and polysorbate among others.

Furthermore, our patient received a tetanus toxoid booster in the form of a Tdap immunization as she was incompletely immunized. Our patient did not receive rabies prophylaxis as the cat was a pet and had never been in the wild, however, rabies prophylaxis should be considered if the injury is inflicted by a wild cat.

\section{Conclusions}

Although cat scratch disease is a common condition that occurs in the pediatric population, cat scratch inflicted corneal injuries are not well described. Performing a detailed eye examination and evaluation of the depth of injury, as well as prompt management are the key for vision rehabilitation. Close follow-up of all cases of corneal injuries is of critical importance for long-term positive outcomes. Rabies prophylaxis should be considered based on the animal exposure.

\section{Acknowledgments}

None to declare.

\section{Financial Disclosure}

Dr. Borkar and Dr. Avendano have disclosed no financial relationships relevant to this article.

\section{Conflict of Interest}

None to declare.

\section{Informed Consent}

Informed consent was obtained from the patient/guardian for the use of data for the purposes of research.

\section{Author Contributions}

Deeksha Borkar carried out the data collection, manuscript writing, preparation and revision of the manuscript; Pablo Avendano conceptualized and designed the case and reviewed and revised the manuscript; and all authors approved the final manuscript as submitted.

\section{Data Availability}

The authors declare that data supporting the findings of this study are available within the article.

\section{References}

1. Browner EA. Corneal abrasions. Pediatr Rev. 2012;33(6):285-286.

2. Malafa MM, Coleman JE, Bowman RW, Rohrich RJ. Perioperative corneal abrasion: updated guidelines for prevention and management. Plast Reconstr Surg. 2016;137(5):790e-798e.

3. Peiris TJ, Khouri AS. Cat-scratch penetrating globe injury with inadvertent fistula and hypotony. Can J Ophthalmol. 2017;52(1):e5-e7.

4. Chang JH, Mills RA, Pater J, Crompton JL. Case series of cat-scratch-inflicted full-thickness corneal lacerations and a review of the literature. Clin Exp Ophthalmol. 2012;40(7):669-674.

5. Campbell TD, Gnugnoli DM. Seidel Test. In: StatPearls. Treasure Island (FL): StatPearls Publishing; 2020.

6. Ahmed F, House RJ, Feldman BH. Corneal abrasions and corneal foreign bodies. Prim Care. 2015;42(3):363-375.

7. Nelson CA, Moore AR, Perea AE, Mead PS. Cat scratch disease: U.S. clinicians' experience and knowledge. Zoonoses Public Health. 2018;65(1):67-73.

8. Griego RD, Rosen T, Orengo IF, Wolf JE. Dog, cat, and human bites: a review. J Am Acad Dermatol. 1995;33(6):1019-1029.

9. Mochizuki Y, Ishikawa H, Sato A, Yamada K, Takesue Y, Gomi F. Pasteurella multocida-induced endophthalmitis after a cat scratch. Am J Ophthalmol Case Rep. 2020;18:100711.

10. Maurin M, Gasquet S, Ducco C, Raoult D. MICs of 28 antibiotic compounds for 14 Bartonella (formerly Rochalimaea) isolates. Antimicrob Agents Chemother. 1995;39(11):2387-2391.

11. Rolain JM, Brouqui P, Koehler JE, Maguina C, Dolan MJ, Raoult D. Recommendations for treatment of human infections caused by Bartonella species. Antimicrob Agents Chemother. 2004;48(6):1921-1933.

12. Goldstein EJ, Citron DM, Merriam CV, Warren YA, Tyrrell K, Fernandez H. Comparative in vitro activity of ertapenem and 11 other antimicrobial agents against aerobic and anaerobic pathogens isolated from skin and soft tissue animal and human bite wound infections. J Antimicrob Chemother. 2001;48(5):641-651.

13. Citron DM, Warren YA, Fernandez HT, Goldstein MA, Tyrrell KL, Goldstein EJ. Broth microdilution and disk diffusion tests for susceptibility testing of Pasteurella species isolated from human clinical specimens. J Clin Microbiol. 2005;43(5):2485-2488.

14. Abrahamian FM, Goldstein EJ. Microbiology of animal bite wound infections. Clin Microbiol Rev. 2011;24(2):231-246.

15. Rabies. Centers for Disease Control and Prevention, Centers for Disease Control and Prevention, Sep. 17, 2019. www.cdc.gov/rabies/index.html.

16. Rossetto O, Scorzeto M, Megighian A, Montecucco C. Tetanus neurotoxin. Toxicon. 2013;66:59-63.

17. Fleisher GR. The management of bite wounds. N Engl J Med. 1999;340(2):138-140. 
18. Ellis R, Ellis C. Dog and cat bites. Am Fam Physician. 2014;90(4):239-243.

19. Domingo E, Moshirfar M, Zabbo CP. Corneal abrasion. In: StatPearls. Treasure Island (FL): StatPearls Publishing; 2020.

20. Robertson SM, Curtis MA, Schlech BA, Rusinko A, Owen GR, Dembinska O, Liao J, et al. Ocular pharmacokinetics of moxifloxacin after topical treatment of animals and humans. Surv Ophthalmol. 2005;50(Suppl 1):S32-45.

21. Miller D. Review of moxifloxacin hydrochloride ophthalmic solution in the treatment of bacterial eye infections. Clin Ophthalmol. 2008;2(1):77-91.

22. Zhang Y, Lu XY, Hu RJ, Fan FL, Jin XM. Evaluation of artificial tears on cornea epithelium healing. Int J Ophthalmol. 2018;11(7):1096-1101. 